\title{
Lamb wave detection with a fiber optic angular displacement sensor
}

Marlon R. Garcia, João M. S. Sakamoto, Ricardo T. Higuti, Cláudio Kitano

Marlon R. Garcia, João M. S. Sakamoto, Ricardo T. Higuti, Cláudio Kitano, "Lamb wave detection with a fiber optic angular displacement sensor," Proc. SPIE 9634, 24th International Conference on Optical Fibre Sensors, 96347I (28 September 2015); doi: 10.1117/12.2195266

SPIE Event: International Conference on Optical Fibre Sensors (OFS24), 2015, Curitiba, Brazil 


\title{
Lamb wave detection with a fiber optic angular displacement sensor
}

\author{
Marlon R. Garcia ${ }^{\mathrm{a}}$, João M. S. Sakamoto ${ }^{\mathrm{b}}$, Ricardo T. Higuti ${ }^{\mathrm{a}}$, Cláudio Kitano*a \\ ${ }^{a}$ Dept. of Electrical Engineering, Universidade Estadual Paulista, Av. Prof. José Carlos Rossi, 1370 \\ Campus III, Ilha Solteira, SP, Brazil 15385-000; ${ }^{\mathrm{b}}$ Div. of Photonics, Instituto de Estudos Avançados, \\ Trevo Cel. Av. José A. A. do Amarante, nº 1, São José dos Campos, SP Brazil 12228-001.
}

\begin{abstract}
In this work we show that the fiber optic angular displacement sensor is capable of Lamb wave detection, with results comparable to a piezoelectric transducer. Therefore, the fiber optic sensor has a great potential to be used as the Lamb wave ultrasonic receiver and to perform non-destructive and non-contact testing.
\end{abstract}

Keywords: Lamb wave, fiber optic sensor, angular displacement, non-destructive testing, non-contact.

\section{INTRODUCTION}

The non-destructive testing (NDT) with ultrasonic waves is a widely used technique ${ }^{1-3}$ in the aeronautics and aerospace industries, for example, for performing flaw detection or measurement of parameters as thickness or porosity. Usually, the generation and the reception of acoustic waves are accomplished by using piezoelectric materials ${ }^{4,5}$. In this work, however, for the detection, we regard the application of a fiber optic angular displacement sensor. This sensor was formerly applied to detection of ultrasonic waves as longitudinal, shear and Rayleigh waves ${ }^{6}$. In the present work, we show the sensor potential for Lamb wave detection in metal plates, which can lead to the development of a non-contact, non-destructive, and coupling medium free technique for NDT with Lamb waves.

\section{FIBER OPTIC ANGULAR DISPLACEMENT SENSOR}

The sensor comprises two single-mode optical fibers: a transmitting and a receiving one, a positive lens, a laser, a photodetector, and a reflective surface. At one end, the fibers are positioned parallel and aligned to each other, in front of the positive lens. This lens is positioned in front of the reflective surface, forming the sensor head shown schematically in Fig. 1. The quantity $Z_{1}$ corresponds to the distance between the positive lens and the reflective surface; $Y_{2}$ corresponds to the position of the spot center (focused at the receiving fiber) in relation to the transmitting fiber center; and $\theta$, to the angular displacement of the reflective surface.

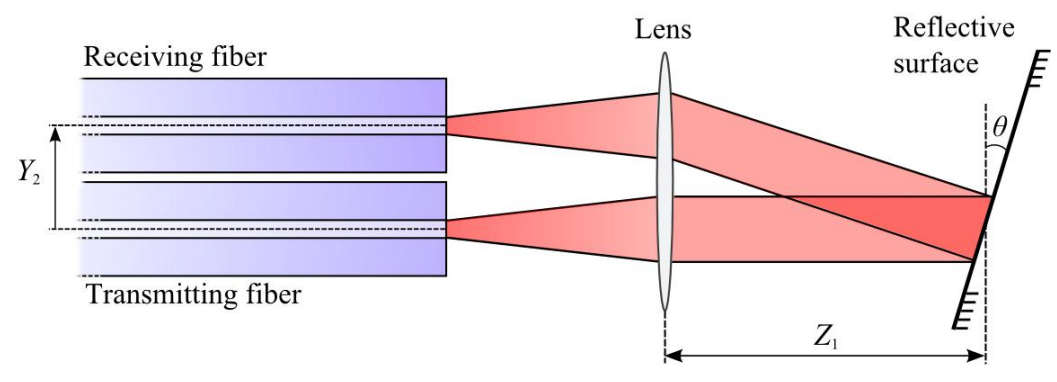

Figure 1. Sensor head.

*kitano@dee.feis.unesp.br; phone +55 183743 1226; fax +55 183743 1150; feis.unesp.br/\#!/dee

24th International Conference on Optical Fibre Sensors, edited by Hypolito José Kalinowski,

José Luís Fabris, Wojtek J. Bock, Proc. of SPIE Vol. 9634, 96347I · c 2015 SPIE

CCC code: $0277-786 X / 15 / \$ 18 \cdot$ doi: $10.1117 / 12.2195266$ 


\subsection{Working principle}

The working principle of the sensor consists in modulating the optical intensity, coupled into the receiving fiber, by the angular displacement of the reflective surface. The optical beam from laser is emitted by the transmitting fiber and collimated by the positive lens up to the reflective surface. After being reflected, the beam passes through the lens again and is focused on the receiving fiber. In this process, the positive lens converts the angular displacement $\theta$ into the spot position, $Y_{2}$, which determines the amount of light coupled into the receiving fiber ${ }^{6}$. Another important function of the lens is to unlink the linear displacement $Z_{1}$ from the angular displacement measurement ${ }^{7}$.

\section{EXPERIMENTAL RESULTS}

In order to verify the potential of the fiber optic angular displacement sensor to detect Lamb waves in an aluminum plate, we mounted an experimental setup, as shown in Fig. 2. The piezoelectric transducer is used to generate a longitudinal wave that, in turn, is coupled to an acrylic prism. This prism allows an incidence angle $\theta_{\mathrm{i}}=30^{\circ}$, which converts the longitudinal wave into the S0 mode of Lamb wave. The parameter $d_{1}$ is the distance that the longitudinal wave travels in the acrylic, while $d_{2}$ is the distance traveled by the Lamb wave in the plate, up to the detector. In the case where the detector is the fiber optic angular displacement sensor, the aluminum plate itself plays the role of the reflective surface.

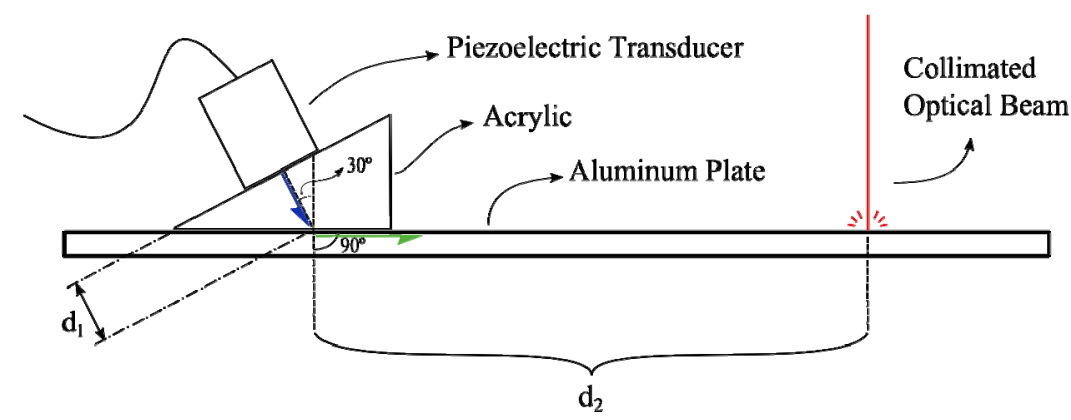

Figure 2. Experimental setup to measure Lamb waves with the fiber optic angular displacement sensor.

The aluminum plate used in this work has dimensions of $300 \mathrm{~mm}$ x $450 \mathrm{~mm} \times 1 \mathrm{~mm}$. It is noteworthy that a slight polishing was applied to the aluminum plate in the region of measurement. In this work, this is needed to the sensor receive a homogeneous light spot and ensure a good signal-to-noise ratio.

Once the plate is positioned, the sensor is aligned in order to determine an operation point (quiescent point) in the middle of the linear region of its characteristic curve. Therefore, angular displacements around the operation point can be detected by the sensor.

The piezoelectric transducer was then driven by a voltage pulse with four cycles and Gaussian envelope at $1 \mathrm{MHz}$ frequency, as shown in Fig. 3.

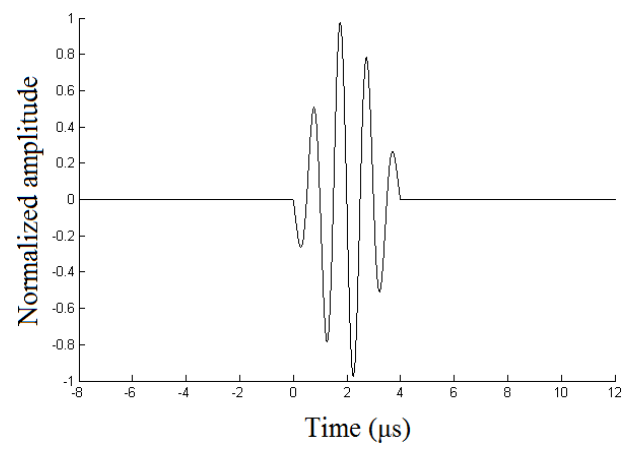

Figure 3. Pulse with four cycles and Gaussian envelope. 
Therefore, the output of the fiber optic angular displacement sensor was then acquired and the result is shown in Fig. 4. The distances $d_{1}$ and $d_{2}$ are $21.59 \mathrm{~mm}$ and $61.94 \mathrm{~mm}$, respectively.

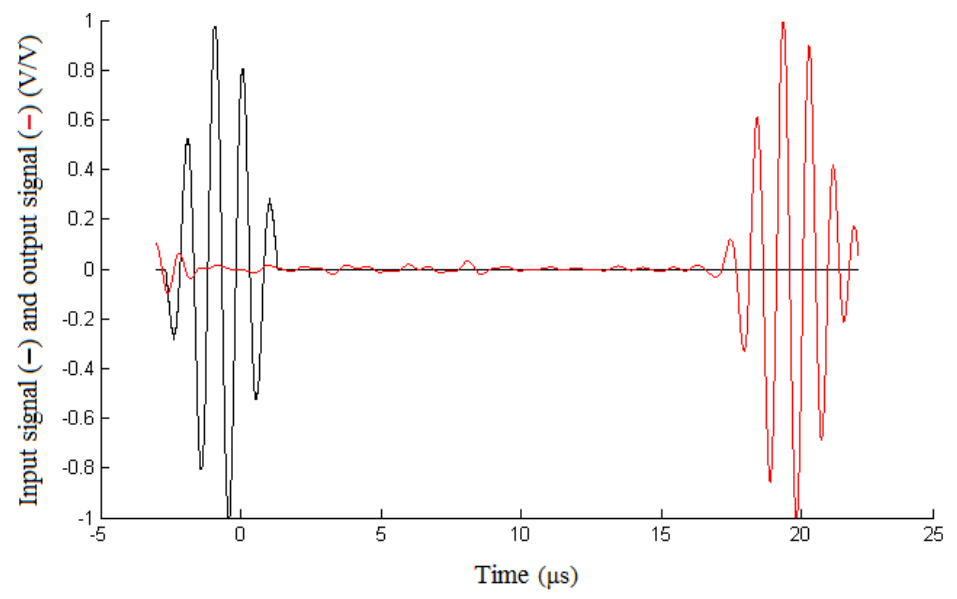

Figure 4. Input signal (black line) and fiber optic angular displacement sensor output (filtered), both normalized.

Using the Hilbert transform, regarding the time difference between the input and the output pulse, the distances $d_{1}$ and $d_{2}$, and the velocity of the longitudinal wave on the acrylic, the velocity of the Lamb wave was measured as $c_{\mathrm{S} 0}=4.953 \mathrm{~mm} / \mu \mathrm{s}$, which presents a difference around $3 \%$ in relation to the $\mathrm{S} 0$ mode theoretical value, $5.091 \mathrm{~mm} / \mu \mathrm{s}$.

Another experiment was performed with a piezoelectric transducer as the detector, in order to compare with the fiber optic sensor performance. In this case, in Fig. 2, the fiber optic sensor's optical beam was replaced by the transducer. The same pulse shown in Fig. 3 was applied to the generating transducer and the corresponding output of the piezoelectric detector was acquired and shown in Fig. 5.

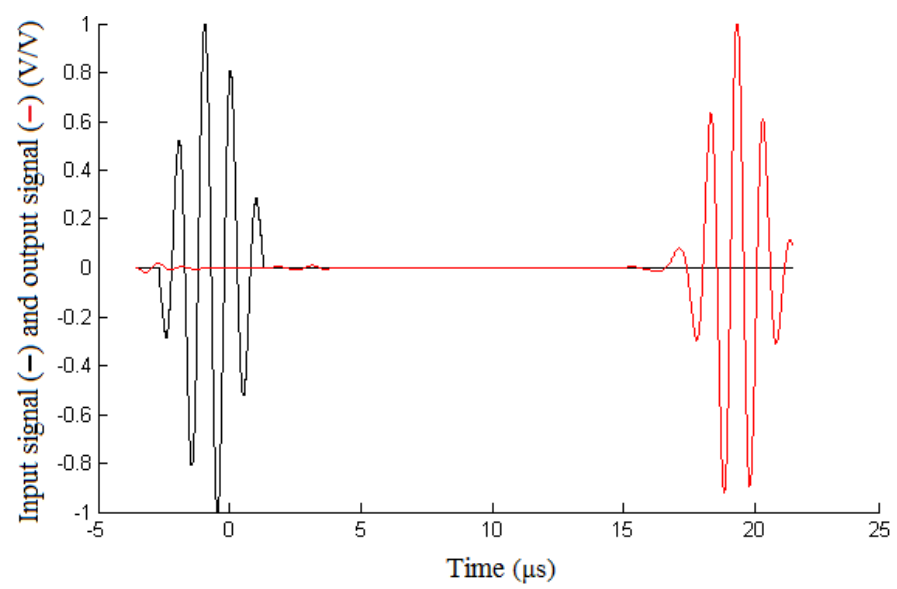

Figure 5. Input signal (black line) and piezoelectric detector output (filtered), both normalized.

The Lamb wave velocity was also measured from the data acquired with the piezoelectric transducer using the Hilbert transform, as $c_{\mathrm{S} 0}=5.203 \mathrm{~mm} / \mu \mathrm{s}$, which means a difference of about $2 \%$ in comparison with the theoretical value. The difference between the fiber optic sensor and the piezoelectric detector was approximately $5 \%$. Figures 4 and 5 present a similar result for the fiber optic angular displacement sensor and the conventional piezoelectric transducer, showing that the fiber optic sensor is capable of detecting Lamb waves. 


\section{DISCUSSION}

In this work we showed that the fiber optic angular displacement sensor is capable of detecting Lamb waves. This means that this sensor has a great potential to perform non-destructive and non-contact inspection by using this type of wave.

The fiber optic angular displacement sensor presents advantages when compared with the piezoelectric transducer, as: mechanical uncoupling with the sample under test, high temperature measurement (e.g. above Curie temperature) is possible, high bandwidth, no mechanical loading is imposed to the structure under test, no coupling medium is required, allows measurement in complex geometry samples, robot compatibility, and allows remote measurement with data transmission by the optical fibers. Among the disadvantages we can cite the following: a reflective surface is needed in the measurement point, higher complexity of the setup, optical alignment is required, and at least by now, it presents lower signal amplitude. The last disadvantage mentioned will be addressed in the next steps in this research.

Once the sensor's potential for detecting Lamb waves was demonstrated, this can lead to the development of a noncontact, non-destructive, and coupling medium free technique for NDT with Lamb waves.

\section{REFERENCES}

[1] Bond, L. J. and Punjani, M., "Review of some recent advances in quantitative ultrasonic NDT," IEE Proc. A: Physical Science, Measurement and Instrumentation, Management and Education, 131 (4), 265-274 (1984).

[2] Ludwig, R. and Lord, W., "A finite-element formulation for the study of ultrasonic NDT systems," IEEE Trans. on Ultrasonics, Ferroelectrics, and Frequency Control, 25 (6), 809-820 (1988).

[3] Lu, H., Wang, Z. and Han, Y., "The ultrasonic NDT technique utilizing the longitudinal or transverse waves induced by Lamb wave in thin plates," IEEE Proc. Instrum. and meas. tech. conf. - IMTC 10, 1195-1197 (1994).

[4] Yanez, Y., Chavez, J. A., Salazar, J., Turo, A. and Garcia-Hemandez, M. J., "Wide dynamic range electronics for air-coupled Lamb-waves ultrasonic NDT using piezoelectric transducer arrays," IEEE Proc. Instrum. and meas. tech. conf. - IMTC 21, 1926-1929 (2004).

[5] Montero de Espinosa, F., Martinez, O., Segura, L. E. and Gomez-Ullate, L., "Double frequency piezoelectric transducer design for harmonic imaging purposes," IEEE Trans. on Ultrasonics, Ferroelectrics, and Frequency Control, 52 (6), 980-986 (2005).

[6] Sakamoto, J. M. S., Kitano, C., Pacheco, G. M. and Tittmann, B. R., "High sensitivity fiber optic angular displacement sensor and its application for detection of ultrasound," Appl. Opt. 51 (20), 4841-4851 (2012).

[7] Sakamoto, J. M. S., Kitano, C., Pacheco, G. M. and Tittmann, B. R., "Geometrical parameter analysis of a highsensitivity fiber optic angular displacement sensor," Appl. Opt. 53 (36), 8436-8443 (2014). 